

\title{
The Benard-Von Karman instability: an experimental study near the threshold
}

\author{
C. Mathis, M. Provansal, L. Boyer
}

\section{To cite this version:}

C. Mathis, M. Provansal, L. Boyer. The Benard-Von Karman instability: an experimental study near the threshold. Journal de Physique Lettres, 1984, 45 (10), pp.483-491. 10.1051/jphyslet:019840045010048300 . jpa-00232372

\section{HAL Id: jpa-00232372 https://hal.science/jpa-00232372}

Submitted on 1 Jan 1984

HAL is a multi-disciplinary open access archive for the deposit and dissemination of scientific research documents, whether they are published or not. The documents may come from teaching and research institutions in France or abroad, or from public or private research centers.
L'archive ouverte pluridisciplinaire HAL, est destinée au dépôt et à la diffusion de documents scientifiques de niveau recherche, publiés ou non, émanant des établissements d'enseignement et de recherche français ou étrangers, des laboratoires publics ou privés. 
Classification

Physics Abstracts

$47.20-47.25 \mathrm{R}-47.30$

\title{
The Benard-Von Karman instability : an experimental study near the threshold
}

\author{
C. Mathis, M. Provansal and L. Boyer \\ Laboratoire de Dynamique et Thermophysique des Fluides, \\ Université de Provence, Centre St Jérôme, 13397 Marseille Cedex 13, France
}

(Reçu le 3 août 1983, révisé le 9 février 1984, accepté le 26 mars 1984)

\begin{abstract}
Résumé. - L'évolution des composantes de la vitesse dans le sillage d'un cylindre est étudiée dans la gamme $40<R e<300$ par anémométrie Laser Doppler à défilement de franges. Le paramètre d'ordre (amplitude de la fluctuation de vitesse perpendiculaire) suit la loi de Landau. L'existence de plusieurs modes est vérifiée et des effets tridimensionnels sont observés et interprétés lorsque deux modes co-existent. La dépendance des grandeurs critiques en fonction du rapport d'aspect est également donnée.
\end{abstract}

\begin{abstract}
The evolution of the velocity components of the wake behind a cylinder has been investigated in the range $40<R e<300$ using a moving fringe Laser Doppler anemometer. The order parameter (amplitude of the perpendicular velocity fluctuations) obeys Landau's law. We confirm the existence of several modes and in particular, we observe and interpret a three-dimensional motion that occurs when two modes co-exist. The dependence of critical quantities on the aspect ratio is also given.
\end{abstract}

\section{Introduction.}

A considerable quantity of work has been devoted to internal flows, for which theoretical models have been developed to explain the behaviour at the instability threshold and far from it [1]. However in the prolific literature concerning external flows where the theoretical approach offers large difficulties, only few studies have been carried out near the threshold. For example to our knowledge there is a stability analysis of an inviscid wake [2], which can give neither the threshold value nor the dependence of the oscillation amplitude as a function of the Reynolds number.

The major features of the flow have been known since the work of Benard [3] or Von Karman [4]. For $R e<4$ the flow simply creeps; at higher values of $R e$ a recirculation zone appears formed by two symmetric eddies attached behind the cylinder. An instability appears at $R e \simeq 40$ in which these eddies are convected away alternately to form a double row of oppositely signed vortices : the Benard-Von Karman vortex street.

Previous numerical and experimental studies have been concerned with measurements of the pressure distribution, the drag the frequency of the vortex-crossing, and to a lesser extent of velocity distributions in the wake. In this paper we present results concerning the properties of the flow in the settled pattern. The results bear mainly on four points : the amplitude of timedependent part of the velocity, the derivation of the "Roshko-Reynolds » relation, the influence of the aspect-ratio, and three-dimensional effects. 


\section{Apparatus.}

This kind of experiment requires very flat velocity profiles and a residual turbulence level as low as possible in the upstream flow. The open-circuit wind-tunnel used here has a 16/1 contraction ratio. The square test-section of size $L=99 \mathrm{~mm}$ is made of plexiglass and is $700 \mathrm{~mm}$ long. The air flow is regulated by sonic throat and calibrated by Laser Doppler Anemometry; the speed ranges from $7.5 \mathrm{~cm} . \mathrm{s}^{-1}$ to $75 \mathrm{~cm} . \mathrm{s}^{-1}$. The velocity profiles are flat with a variation of less than $\pm 1 \%$ over $L_{\mathrm{e}}=70 \mathrm{~mm}$ of the test section and the free-stream turbulence is less than $0.3 \%$. The eleven cylinders used in this work are made of rectified and polished steel. The diameters $d$ are known with an accuracy of $0.5 \%$ and range from $1.3 \mathrm{~mm}$ to $20 \mathrm{~mm}$. When necessary we have taken into account the flow acceleration produced by a low blockage ratio. In our experiments, the blockage ratio is equal to the aspect ratio $L / d$ due to the square section of the wind-tunnel. The optical part of the laser anemometer including the frequency shifter based on a rotating radial diffraction grating [5] was built in the laboratory. The technique of frequency shifting is necessary whenever the velocity changes sign as is the case for $U_{y}$ and $U_{z}$ The frequency shifter was designed to produce an apparent velocity shift which could be adjusted in the range $+10 \mathrm{~cm} \cdot \mathrm{s}^{-1}$ to $+30 \mathrm{~cm} \cdot \mathrm{s}^{-1}$. This was compatible with the oscillating transverse velocity that had a maximum amplitude of $20 \mathrm{~cm} . \mathrm{s}^{-1}$. The spatial resolution is $100 \mu \mathrm{m}$ and the accuracy is about $0.3 \%$. The streaklines have been visualized by local seeding associated with laser tomography. The sheet of laser light could be rotated to visualize any desired axial cut of the test section. The smoke was injected via $0.5 \mathrm{~mm}$ holes in the walls of hollow cylinders. These cylinders were limited to the range $6 \mathrm{~mm}$ to $10 \mathrm{~mm}$. The Doppler Signal is processed by a TSI counter model 1980 and the analogic output, proportional to the speed, is analysed in real time (analyser Rockland F.F.T. 512 S). The mean sampling frequency is about $2500 \mathrm{~Hz}$; the analogic signal, low pass filtered, can then be considered as a good representation of the velocity for frequencies up to around $50 \mathrm{~Hz}$. In all cases the measurements are made at the point $(5 d, 0,0)$ (Fig. 1$)$ to avoid any boundary effects.

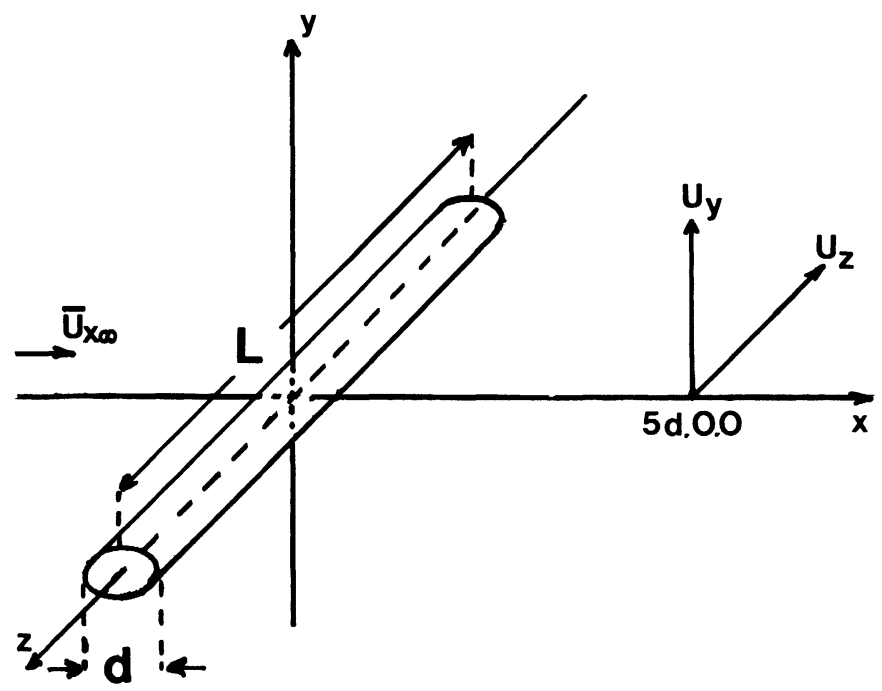

Fig. 1. - Definition of the geometry.

\section{Results.}

3.1 OSCILlation THRESHOLD. - As for a duct flow, the critical value of Reynolds number depends on a number of parameters : the aspect ratio $L / d$, the shear stress rate and the freestream 
turbulence level. Different authors advance values distributed over the range $34<\operatorname{Re}<48$ [610]. Shair et al. [11] have plotted different values of $R e_{\mathrm{c}}$ versus aspect ratio $L / d$ - the extrapolated value for infinite $L / d$ yields Roshko or Kovasznay's value $: R e_{\mathrm{c}}=40$. A more complete and recent set of measurements made by Nishioka and Sato [10] leads to the limiting value : $R e_{\mathrm{c}}=48$. We have observed, in agreement with the previously quoted authors, a variation of $R e_{\mathrm{c}}$ with the aspect ratio. Corrected values $R e_{\mathrm{c}}^{\mathrm{cc}: \mathrm{r}}$ have been calculated to take into account the finite size of the test section when the blockage ratio $L / d$ is not very large. In fact, an effective length $L_{\mathrm{e}}$ equal to the width of the velocity profile is used instead of $L: R e_{\mathrm{c}}^{\mathrm{corr}}=R e_{\mathrm{c}}\left(1-\frac{d}{L_{\mathrm{e}}}\right)^{-1}$ (Fig. 2). Our experimental values of $R e_{\mathrm{c}}^{\text {corr }}$ are well represented by the following cubic expression :

$$
R e_{\mathrm{c}}^{\mathrm{corr}}=47.0+144\left(\frac{d}{L}\right)+1737\left(\frac{d}{L}\right)^{2}+3264\left(\frac{d}{L}\right)^{3}
$$

with

$$
0.013<\frac{d}{L}<0.202
$$

within the accuracy of the measurements $(1 \%$ for $R e)$, the limiting value (for $L / d$ infinite) $R e_{\mathrm{c}}^{\text {corr }}=$ 47.0 is in good agreement with the one advanced by Nishioka and Sato.

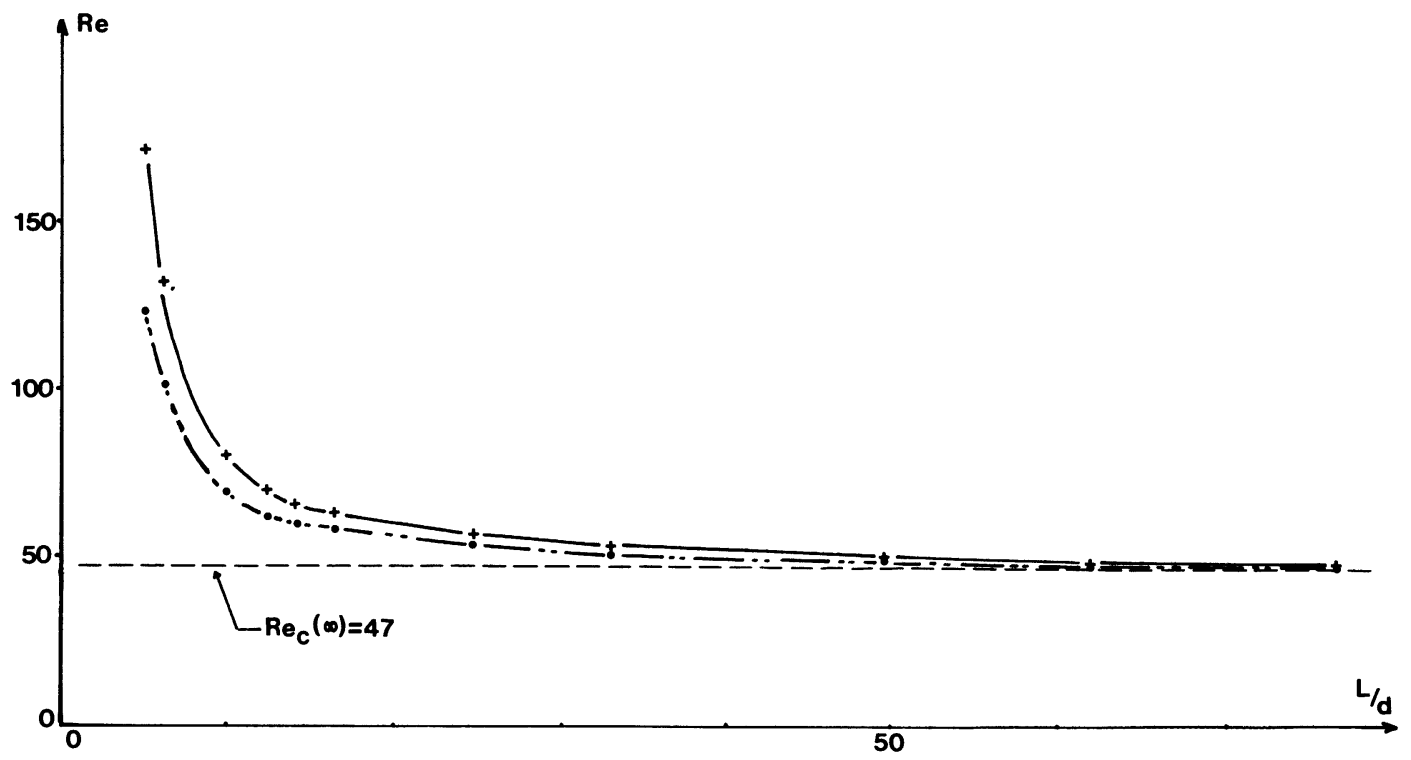

Fig. 2. - Critical Reynolds number versus aspect ratio. $\bullet$ : Non corrected values; + : Corrected values.

3.2 THE TRANSVERSE VelOCITY, ORDER PARAMETER OF THE TRANSITION. - Figure 3 shows, for three of the cylinders, the square of the transverse amplitude velocity $\left(U_{\mathrm{y}}^{0}\right)^{2}$ as a function of $(\operatorname{Re}-$ $\left.R e_{\mathrm{c}}\right)$, on a logarithmic plot : the critical exponent associated with the amplitude is $0.50 \pm 0.01$ for all of the eleven cylinders investigated. We may deduce that the Benard-Von Karman instability is 


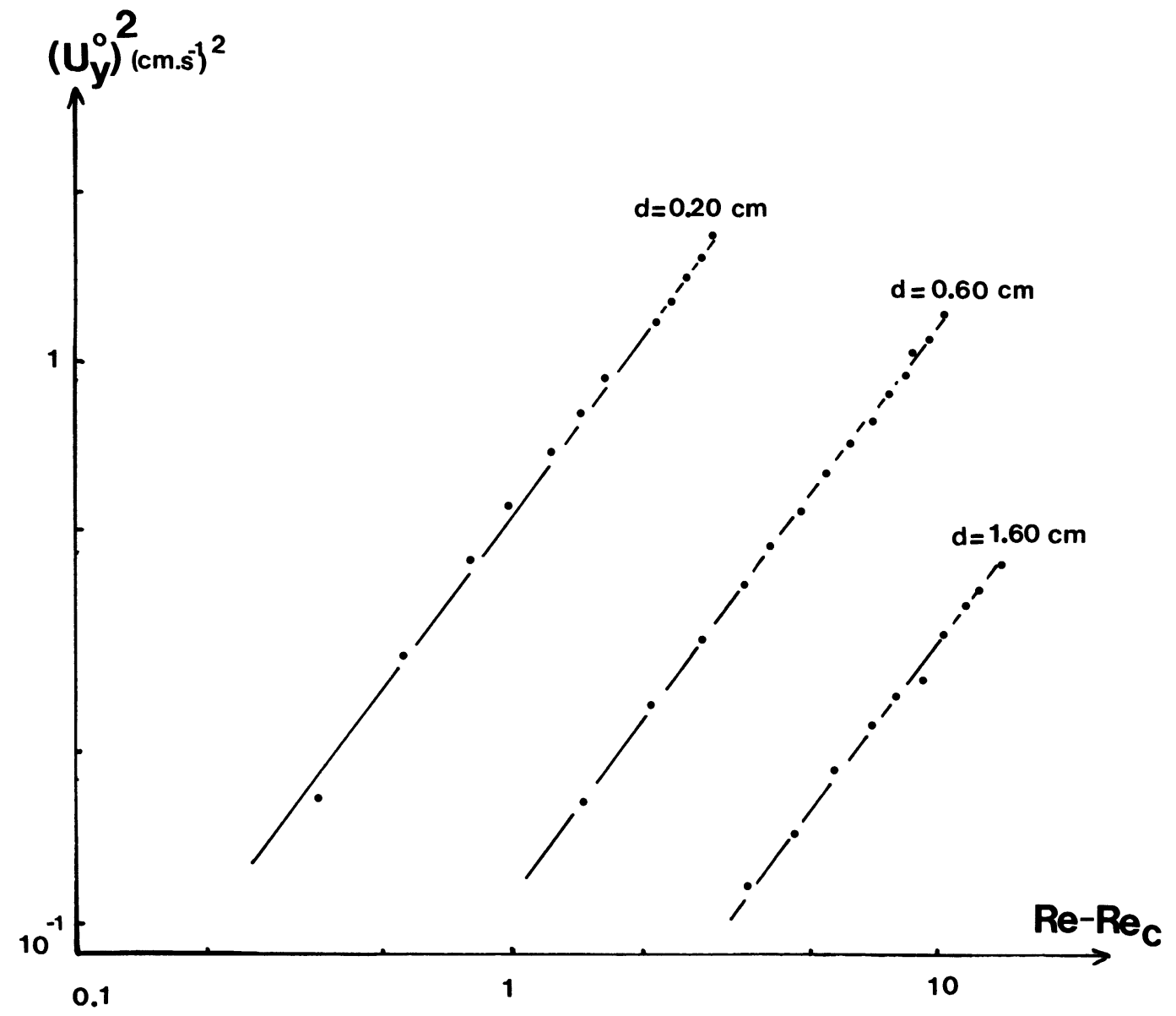

Fig. 3. - Transverse kinetic energy versus $\left(R e-R e_{\mathrm{c}}\right)$. In a $\log -\log$ plot, a linear regression gives a slope equal to :

$-d=0.20 \mathrm{~cm}: 1.01 \pm 0.01$

$-d=0.60 \mathrm{~cm}: 1.01 \pm 0.01$

$-d=2.00 \mathrm{~cm}: 0.98 \pm 0.01$.

characterized, to the present accuracy of measurements, by a direct bifurcation and can be described by the real part of the Landau equation [12-13] :

$$
\frac{\mathrm{d} U_{\mathrm{y}}}{\mathrm{d} t}=\left(\sigma_{\mathrm{R}}+i \sigma_{\mathrm{I}}\right) U_{\mathrm{y}}-\frac{1}{2}\left(l_{\mathrm{R}}+i l_{\mathrm{I}}\right)\left(U_{\mathrm{y}}^{0}\right)^{2} U_{\mathrm{y}} \text { with } U_{\mathrm{y}}=U_{\mathrm{y}}^{0} \mathrm{e}^{i \phi(t)}
$$

For any cylinder the velocity amplitude is given by :

$$
U_{\mathrm{y}}^{0}\left(\mathrm{~cm} . \mathrm{s}^{-1}\right)=0.15\left(\frac{L_{L}}{d}\right)^{0.70}\left(R e-R e_{\mathrm{c}}\right)^{0.50}
$$

which is the steady solution to Landau equation for the amplitude where $\sigma_{\mathrm{R}}=k\left(\operatorname{Re}-\operatorname{Re} e_{\mathrm{c}}\right)$. 
In dimensionless form :

$$
R e_{\mathrm{osc}}=\operatorname{Re}_{L}\left(\frac{L}{d}\right)^{-0.30}\left(R e-R e_{\mathrm{c}}\right)^{0.50}
$$

where $\left\{\begin{array}{l}\operatorname{Re}_{\mathrm{ox}}=\frac{U_{\mathrm{y}}^{0} d}{v} \\ \operatorname{Re}_{\mathrm{L}}=\frac{0.15 L}{v}\end{array}\right.$ and $v$ is the kinematic viscosity.

The coefficient 0.15 is a typical value of the location $(5 d, 0,0)$ investigated. Further experiments are undertaken to study the spatial variation of the amplitude.

The dependence $(L / d)^{-0.3}$ describes our experiments where the aspect ratio is in the range $4.96<L / d<76.3$.

3.3 The Roshko-Reynolds Relation IN THE LANDAU MODEL. - From dimensional analysis, two dimensionless frequencies can be built up, which are the Strouhal number $S t=f d / \bar{U}_{\mathrm{x} \infty}[14]$ and the Roshko number $R o=S t R e=f d^{2} / v$ [8]. The well known linear relation $R o=a R e+b$ can be deduced from the imaginary part of the Landau equation :

$$
\frac{\mathrm{d} \phi}{\mathrm{d} t}=\sigma_{\mathrm{I}}-\frac{1}{2} l_{\mathrm{l}}\left(U_{\mathrm{y}}^{0}\right)^{2}
$$

where $\frac{1}{2 \pi} \frac{\mathrm{d} \phi}{\mathrm{d} t}=f:$ shedding frequency

and $\frac{1}{2 \pi} \sigma_{\mathrm{I}}=f_{\mathrm{c}}:$ shedding frequency at the threshold.

It follows that $R o=a R e+b$ with $a=-\frac{1}{2 \pi} k \frac{d^{2}}{v} \frac{l_{I}}{l_{\mathrm{R}}}$.

It is thus evident that the « Roshko-Reynolds » relation is also compatible with a Landau model.

3.4 THE Two OSCILlating MODES. - For the low aspect-ratio cylinders $(L / d \leqslant 12.4)$ the " $R o / R e$ » relation describes well the experimental results up to $R e \simeq 300$.

However the higher aspect-ratio cylinders do present a change of mode apparently of the same nature as the one observed by Tritton in 1959 [15-16] and also by Berger and Wille [17], and which has been discussed in a long debate by Gaster [18-19] without conclusion.

Figure 4 shows the variation of $R o$ and $\left(U_{y}^{0}\right)^{2}$ versus $R e$, experimentally observed for the $6 \mathrm{~mm}$ diameter cylinder, and we can distinguish three zones : the a and c zones where the frequency is characterized uniquely by a single line in the power spectrum and the $b$ zone which presents two incommensurate frequencies characterizing the two modes. In this zone the energy decreases by 6 or $7 \mathrm{~dB}$ before increasing once again in the $\mathrm{c}$ zone : This property had been already observed but less clearly by Tritton [15].

We have also investigated $U_{z}(t)$ - the velocity component parallel to the axis of the cylinder. $U_{\mathrm{z}}(t)$ is almost zero in the a zone $:\left(U_{\mathrm{z}}^{0}\right)^{2} /\left(U_{\mathrm{y}}^{0}\right)^{2} \simeq 10^{-2}$. The energy associated with $U_{\mathrm{z}}(t)$ increases suddenly and strongly in the biperiodic regime (b zone) and exhibits once again a smooth growth in the $\mathrm{c}$ zone. In the $\mathrm{b}$ regime this signal has a characteristic saw-tooth shape (Fig. 5a). Its power spectrum shows a low frequency line equal to the difference of frequencies of the two modes.

There is a simultaneous evolution of $U_{z}(t)$ and of the vortex lines observed by the light sheet in the $x-z$ plane (Fig. 5b). The vortex lines have a progressive but strong bending ( $\alpha$, Fig. 5a) followed by a fast jump ( $\beta$, Fig. 5a) to the primitive state, where the vortex lines are parallel to the cylinder. 

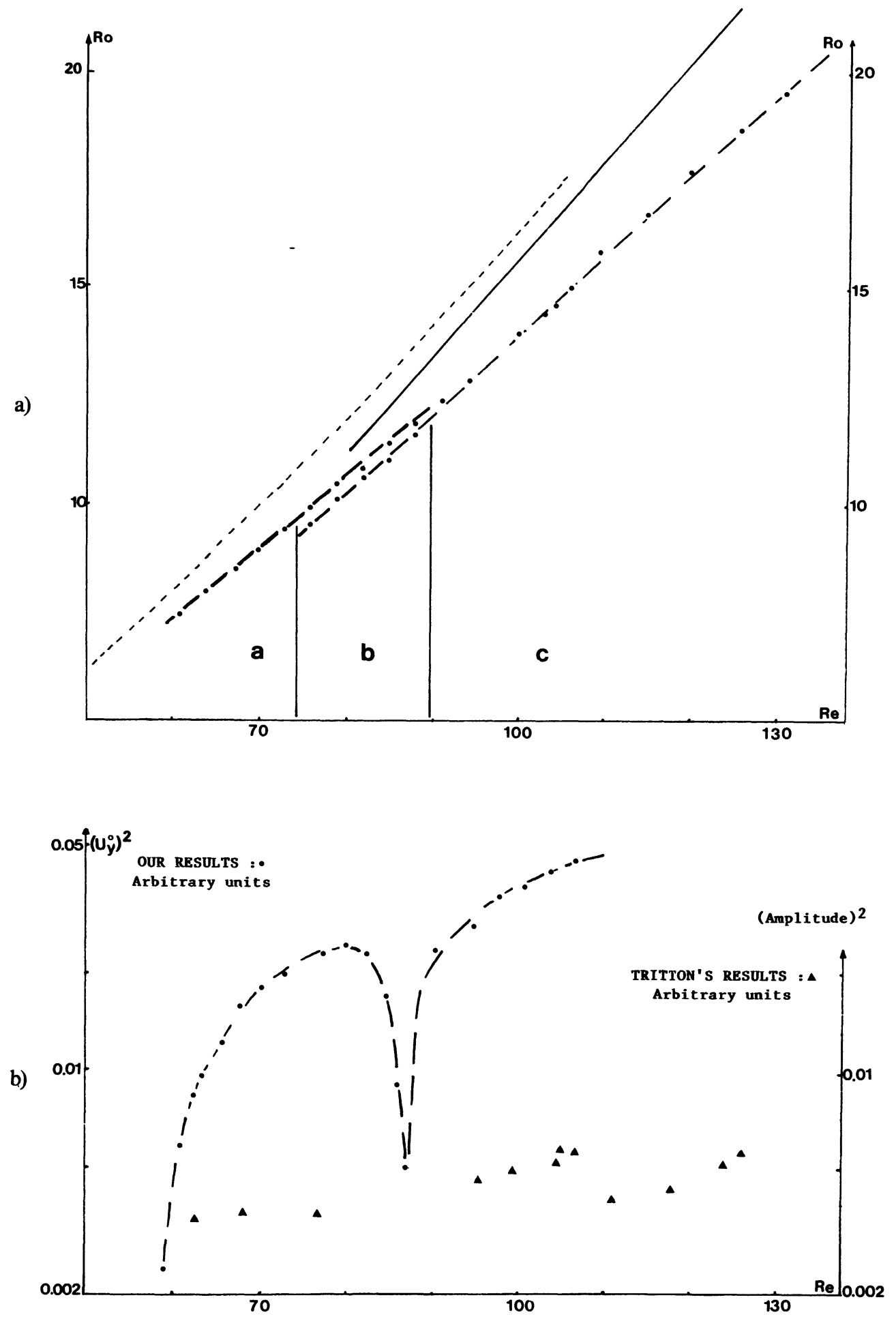

Fig. 4. - a) The two oscillating modes : Frequency versus Re. Our results -- - ; Tritton's low speed mode - - -; Tritton's high speed mode — - b) The two oscillating modes : Energy versus Re. In our experiments $d=0.60 \mathrm{~cm}$; in Tritton's experiments $d=0.178 \mathrm{~cm}$. 


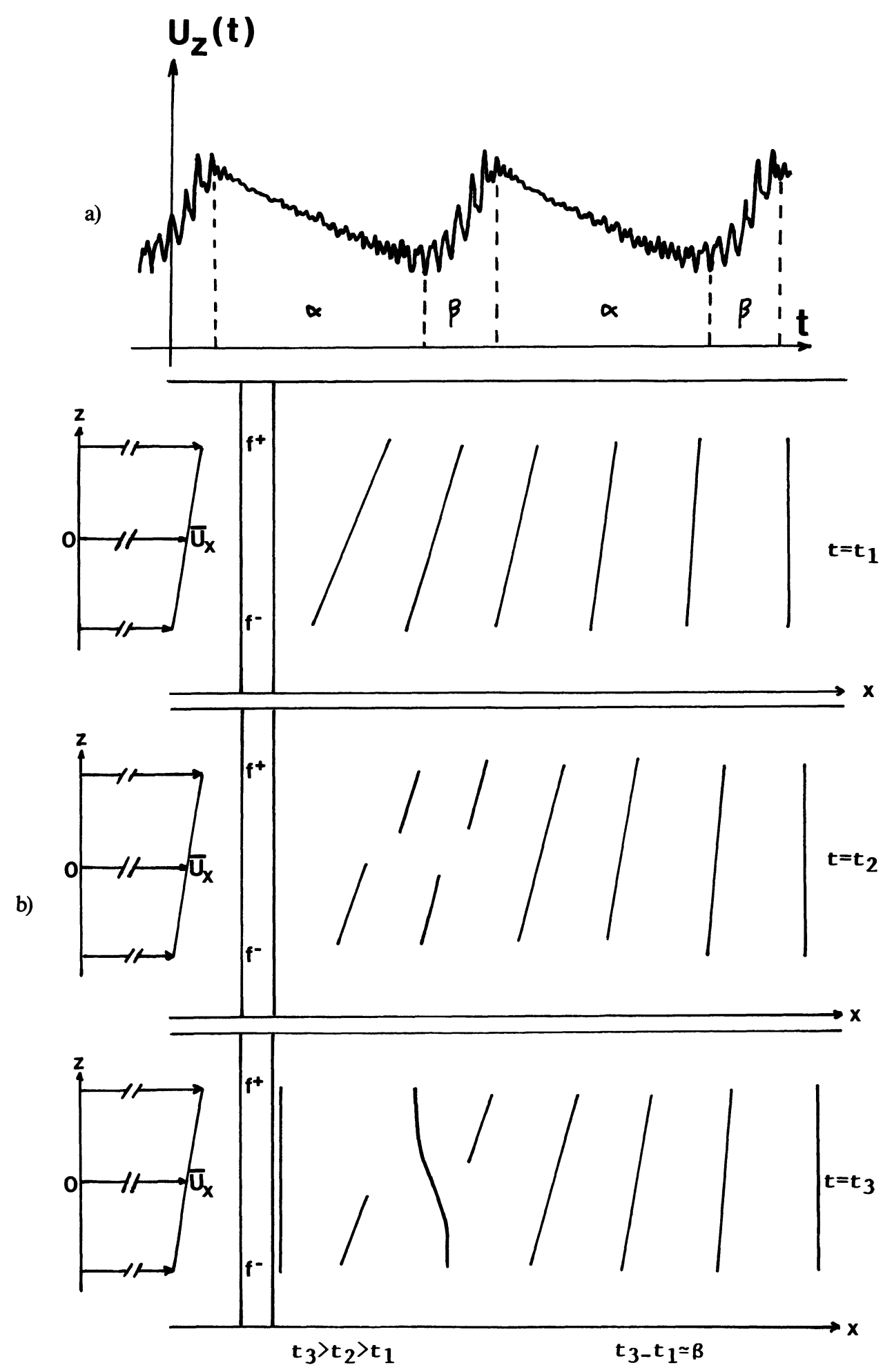

Fig. 5. - a) The saw-tooth behaviour of $U_{z}(t)$. b) Sketch of the time evolution of the vortex lines. (Photographs are of too poor quality to be reproduced here.) 
We may also remark that the " $R o / R e$ » relation always has a higher slope in the second mode despite the similarity in flow-pattern seen through streakline observations. Moreover the Reynolds number $R e_{\mathrm{M}}$, for which the second mode appears, tends to $R e_{\mathrm{c}}$ when $L / d$ increases : This behaviour is well described by

$$
\frac{R e_{\mathrm{M}}}{R e_{\mathrm{c}}}=1+55\left(\frac{L}{d}\right)^{-1.80} \text { for } \quad \frac{L}{d} \geqslant 14.2 .
$$

In addition, a change of sign of the velocity gradient $\Delta \bar{U}_{\mathrm{x} \propto} / \bar{U}_{\mathrm{x} \infty}$ produces a change of bending of the vortex lines. Therefore the growth of the second mode is due both to a high aspect ratio and a residual velocity gradient. Indeed, differentiation of the dimensional form of the " $R o / R e$ " relation gives :

$$
f=\frac{v}{d^{2}}\left[\frac{\Delta \bar{U}_{\mathrm{x} \infty}}{\bar{U}_{\mathrm{x} \infty}} a R e-\frac{\Delta d}{d}(a R e+2 b)\right] .
$$

In the present experiments, $\Delta f \simeq \frac{a}{L} \Delta \bar{U}_{\mathrm{x} \infty} \frac{L}{d}$. Gasters' phenomenological interpretation is confirmed here and his results completed. We also mention the observation of Gerrard [20] on three dimensional effects which he attributes to a " variation of velocity magnitude and direction along the cylinder length $»$.

\section{Conclusion.}

This study reports new results concerning the nature of the Benard-Von Karman instability near the threshold and the dynamics of the wake. In the steady periodic regime the Landau law on the amplitude of the order parameter is verified - with good accuracy. Moreover, the Landau equation for the phase allows us to deduce the " $R o / R e$ » relation. The next step is the investigation of the transient and forced regimes to provide a more complete check of the Landau equation; such work is in progress.

The two modes quoted by some authors (Tritton, Berger, ...) have been clearly observed with distinct slopes. Moreover, in the coexistence range a three-dimensional motion occurs. The defects of the velocity profile are responsible for the symmetry breaking but it seems that this spanwise oscillation is an intrinsic mechanism. As a matter of fact it vanishes for a fixed Reynolds number, the system oscillating again in a pure mode. Further experiments are planned : Some are related to the three-dimensional effects (tapered cylinders, shear flow, several points measurements), other ones to the interesting problem of the growth of the recirculation zone.

\section{Acknowledgments.}

We are grateful to Y. Pomeau and A. Liñan for stimulating discussions. We also thank F. Abetino and J. P. Pahin for their technical assistance. We are indebted to D. Jeandel who calculated the contraction. This work is supported by C.N.R.S. and the M.I.R. 


\section{References}

[1] Bergé, P., Dynamical and Critical Phenomena (Springer Verlag Genève) 1979.

[2] Betchov, R. and Criminale, Jr W. O., Phys. Fluids 9-2 (1966) 359.

[3] Bénard, H., Comp. Rend. 147 (1908) 839.

[4] Von Karman, Th., Gött. Nachr. 509 (1911).

[5] Stevenson, W. H., Appl. Opt. 9 (1970) 649.

[6] Richardson, Modern Developments in Fluid Mechanics, vol. II, S. Goldstein, Editor (Dover Pub.) 1965.

[7] Kovasznay, L. S. G., Proc. R. Soc. A 198 (1949) 174.

[8] Roshko, A., N.A.C.A. Report (1954) 1991.

[9] Collis, D. C. and Williams, M. J., J. Fluid Mech. 6 (1959) 357.

[10] NishioKa, M. and SATO, H., J. Fluid Mech. 65 (1974) 97.

[11] Shair, F. N., Grove, A. S., Peterson, E. E. and Acrivos, A., J. Fluid Mech. 17 (1963) 546.

[12] Landau, L. D. and Lifshitz, E. M., Mécanique des Fluides (Ed. Mir, Moscou) 1971.

[13] Landau, L. D. and Lifshitz, E. M., Physique Statistique (Ed. Mir, Moscou) 1967.

[14] Lord RAYLeIGH, J. W. S., The theory of sound (Dover Publication New York), 1945, vol. 2, p. 412.

[15] Tritton, D. J., J. Fluid Mech. 6 (1959) 547.

[16] Tritton, D. J., J. Fluid Mech. 45-1 (1971) 203.

[17] Berger, E. and Wille, R., Annu. Rev. Fluid Mech. 4 (1972) 313.

[18] GASTER, M., J. Fluid Mech. 38-3 (1969) 565.

[19] GASTER, M., J. Fluid Mech. 46 (1971) 749.

[20] Gerrard, J. H., J. Fluid Mech. 25-1 (1966) 143. 